\title{
Mechanical Fragility of Erythrocyte Membrane in Neonates and Adults
}

\author{
THOMAS BÖHLER, ALBRECHT LEO, ACHIM STADLER, AND OTWIN LINDERKAMP \\ Division of Neonatology, Department of Pediatrics, University of Heidelberg, Heidelberg, Germany
}

\begin{abstract}
The shortened life span of neonatal red blood cells (RBC) is associated with accelerated membrane loss. The present study was designed to measure the critical shear force that causes membrane failure and the rate of membrane failure for neonatal and adult RBC. A micropipette technique was used to determine the membrane extensional (shear) elastic modulus (i.e. resistance of the membrane to extensional elastic deformation), the rate of extensional membrane deformation (i.e. surface viscosity), and the tension for local membrane fragmentation. A flow channel system was used to determine the critical shear force of plastic membrane deformation (i.e. beginning of membrane tether formation), the rate of plastic deformation, and the plastic shear viscosity coefficient. The extensional elastic modulus of neonatal RBC was $18 \%$ smaller and the rate of elastic deformation was $25 \%$ longer compared with adult cells $(p<0.05)$. Membrane surface viscosity was similar for both cell types. The tension for local membrane fragmentation in the micropipette was $23 \%$ lower in neonates than in adults. However, the strain (i.e. extent of membrane deformation calculated as ratio of the stress resultant and the elastic modulus) at which membrane rupture in the micropipette occurred was similar for neonatal and adult RBC. This indicates that the smaller critical tension for neonatal RBC membrane failure was due to increased membrane elastic deformability. The critical shear force for membrane tether formation in the flow channel was similar for neonatal and adult RBC (1.17 \pm 0.13 vs $1.19 \pm 0.21 \times 10^{-6}$ dyne, $\left.p>0.1\right)$, whereas the tether growth was about two times faster and the plastic viscosity coefficient was $\mathbf{5 0 \%}$ less for neonatal RBC compared with adult cells. These results indicate that the critical force for membrane failure is similar for neonatal and adult $\mathrm{RBC}$ if the increased membrane deformability of neonatal RBC is considered. However, once the membrane begins to fail, neonatal RBC appear to lose more membrane per unit time. (Pediatr Res 32: 92-96, 1992)
\end{abstract}

\section{Abbreviations}

RBC, red blood cell

The mean life span of neonatal RBC is about $60 \mathrm{~d}$, which is one-half that of normal adult cells (1). During the physiologic aging process, $\mathrm{RBC}$ lose membrane fragments and water, leading to a decrease in surface area, volume, and deformability and an increase in density and mean cell $\mathrm{Hb}$ concentration (2-4).

Neonatal RBC show an accelerated loss in volume, surface

Received August 21, 1991; accepted January 20, 1992.

Correspondence and reprint requests: Dr. T. Böhler, Universitäts-Kinderklinik Heidelberg, Im Neuenheimer Feld 150, D-6900 Heidelberg, Germany.

Supported by a research grant (Li 291/4-3) from the Deutsche Forschungsgemeinschaft. area, and deformability compared with adult $\operatorname{RBC}(5,6)$. Early damage and shortened life span of neonatal RBC may be explained by several peculiar RBC properties: 1) rapid decline of intraerythrocytic enzyme activities and the ATP level $(7,8) ; 2)$ increased susceptibility of membrane lipids and proteins and of $\mathrm{Hb}$ to peroxidation $(9,10) ; 3)$ loss of membrane surface area by endocytosis and internalization of membrane lipids $(6,11)$; and 4 ) increased mechanical fragility of neonatal RBC (12-14). Increased mechanical fragility of neonatal $\mathrm{RBC}$ has been shown by subjecting cell suspensions to extremely high shear forces using glass beads $(12,13)$ and an ultrasound technique (14). However, mechanical fragility studied by these techniques increases with increasing RBC membrane elasticity and with decreasing cell density (14); neonatal RBC show both increased membrane elasticity $(15)$ and decreased cell density $(16,17)$ compared to adults.

Mechanical rupture and fragmentation of $\mathrm{RBC}$ membrane is principally a result of extreme elastic membrane deformation. The $\mathrm{RBC}$ membrane deforms in response to a deforming force until the yield point of the membrane material is reached. Beyond this point, additional deforming forces result in irreversible plastic deformation of the membrane. Membrane deformation and fragmentation can be characterized by several mechanical material properties $(18,19): 1)$ the shear (extensional) elastic modulus defines the resistance of the membrane to elastic (i.e. reversible) deformation; 2) the shear (surface) viscosity describes the rate (i.e. time-dependency) of elastic membrane deformation; 3 ) the critical shear force defines the deforming shear force that causes plastic deformation (i.e. membrane failure); and 4) the plastic viscosity coefficient characterizes the rate of membrane failure.

The present investigation was designed to study membrane fragility of individual neonatal and adult RBC using two microscopic techniques: 1) A micropipette technique was used to determine the membrane shear elastic modulus, the shear viscosity, and the critical tension for local fragmentation of the same cell; and 2) a flow channel system was used to determine the critical shear force of plastic membrane deformation (i.e. beginning of membrane tether formation) and the plastic viscosity coefficient.

\section{MATERIALS AND METHODS}

Blood samples and $R B C$ preparation. Placental blood samples from 16 newborn infants were studied with the approval of the Department of Pediatrics Human Subjects Research Committee. They were healthy, full-term infants with gestational ages of 38 to $40 \mathrm{wk}$ and birth weights of 3210 to $3640 \mathrm{~g}$. Infants with malformations, erythroblastosis, diabetic mothers, hemorrhage, or cesarean section were excluded, as were twins and infants with proven infection or high risk of infection. The umbilical artery pH was above 7.25, the 1-min Apgar score was 9, and the 2- and 5-min Apgar scores were 10 in all cases. All infants had birth weights appropriate for gestational age (10th-90th percentile according to Munich charts) $(20)$. Blood (5-10 mL) was collected 
from the placenta into EDTA (approximately $1 \mathrm{mg} / \mathrm{mL}$ ) immediately after cord clamping before delivery of the placenta. Adult blood samples $(10 \mathrm{miL})$ were collected from 15 healthy male laboratory personnel (aged 21 to $35 \mathrm{y}$, nonsmokers, not obese) by venipuncture into EDTA. Placental blood from six neonates and venous blood from five male adults was used for the flow channel measurements; 10 neonatal and 10 adult blood samples were collected for the micropipette studies.

Blood samples were stored on ice during the transport to the laboratory. All measurements were made at room temperature $\left(23 \pm 1^{\circ} \mathrm{C}\right)$ within $4 \mathrm{~h}$ after collection. Part of each blood specimen was not centrifuged and washed before dilution in the appropriate suspending medium, because neonatal RBC are sensitive to morphologic transformation and mechanical damage. Another portion of each blood sample was centrifuged to obtain plasma. A solution of PBS $\left(0.005 \mathrm{~mol} / \mathrm{L} \mathrm{KH}_{2} \mathrm{PO}_{4}+\right.$ $\mathrm{Na}_{2} \mathrm{HPO}_{4}, 0.153 \mathrm{~mol} / \mathrm{L} \mathrm{NaCl}, \mathrm{pH} 7.40,294 \mathrm{mosmol} / \mathrm{kg}$ ) containing $1 \mathrm{~g} / \mathrm{L}$ human serum albumin (Behring, Marburg, Germany) was used as suspending medium for RBC (final hematocrit $0.01-0.02$ ). All solutions were filtered through $0.2 \mu \mathrm{m}$ Sterivex filters (Millipore Corp., Bedford, MA) before use.

Flow channel. We determined the critical shear force $\left(\mathrm{F}_{\mathrm{c}}\right)$ and the plastic viscosity coefficient $\left(\eta_{\mathrm{P}}\right)$ in a parallel-plate flow channel system that consists of the flow channel, an inverted microscope (Leitz Diavert, Wetzlar, Germany), a Harvard variable speed DC infusion pump (Harvard Apparatus Co., Inc., Millis, MA), and a video system. The Harvard pump was used to pump PBS solution through the flow channel via a gas-tight precision syringe (Hamilton, Bonadenz, Switzerland). The pressure drop in the channel was monitored with a piezo-resistive pressure transducer (sensor: model SN R 16, Druckmeßtechnik Keller AG, Winterthur, Switzerland). The microscope was equipped with a water-immersion objective (50×, planar objective 50/1.0) and a $2 \times$ optical lens converter. The optical magnification of the microscope was $788 \times$. The additional electronic magnification of the video system resulted in a final magnification of $4000 \times$. The video system consisted of a $1 / 2$-inch $C C D$ video camera (Panasonic WV CD 110 E; Matsushita Electric, Tokyo, Japan), a video tape recorder (Panasonic AG 6200 EG, Matsushita Electric), a 14-inch video monitor (Panasonic TC 431 DRP, Matsushita Electric), a video-timer (VTG-33; For-A, Tokyo, Japan), and a contour synthesizer (IV-530, For-A). The evaluation of the video recordings was done using an image analyzing system (MOP Videoplan; Kontron, Eching, Germany). The video picture was calibrated with a micrometer (calibration bars of $20 \pm 0.2 \mu \mathrm{m}$ ) in an overlay of the video analyzing system.

The flow channel was constructed as described by Hochmuth et al. (21) and Waugh (22). A narrow channel was formed using a glass coverslip and a Plexiglas holder separated by a $0.12-\mathrm{mm}$ thick Teflon layer. Fluid passed through the channel by inlet and outlet holes in the Plexiglas holder. The distance between the holes was $40 \mathrm{~mm}$ and the width of the channel was $8.85 \mathrm{~mm}$. One of the holes was connected to the Harvard pump by means of silicone tubing. The flow channel was initially filled with $10 \%$ autologous plasma diluted in PBS. This procedure prevents adhesion of a large RBC surface area to the glass. The plasma was flushed out, and the channel was filled with the RBC suspension. The RBC were allowed to settle for $20 \mathrm{~min}$. The chamber was then cleared of unattached cells; only cells attached to a single point were selected for observation. After this, PBS containing $1 \mathrm{~g} / \mathrm{L}$ of human serum albumin was infused into the channel at a low flow rate and the cells were observed and videorecorded for approximately $1 \mathrm{~min}$. The flow was stopped and the flow direction was reversed to check for more than one attachment point of an RBC to the coverslip. Then the flow rate was increased. The cell was again observed for $1 \mathrm{~min}$. This process was repeated until the cell became detached from the glass. Only cells with four or more measurements at increasing flow rates were included in the final analysis. The resulting wall shear stresses $\left(\mathrm{T}_{\mathrm{w}}\right)$ ranged from 1.0 to 12.0 dyne $/ \mathrm{cm}^{2}$.
$T_{w}$ is related to the pressure drop $\Delta P$ :

$$
\mathrm{T}_{\mathrm{w}}=\Delta \mathrm{P} \cdot \mathrm{H} / 2 \mathrm{~L}
$$

where $\mathrm{H}$ is the height (approximately $0.12 \mathrm{~mm}$ ) and $\mathrm{L}$ is the length (approximately $40 \mathrm{~mm}$ ) of the channel.

The shear force $\left(F_{s}\right)$ acting on the cells attached to the channel wall was calculated from $T_{w}$ and the projected area of the cell $\left(A_{p}\right)$ :

$$
F_{s}=T_{w} \cdot A_{p}
$$

$A_{p}$ was calculated from the radius ( $\left.r\right)$ of the resting cell $\left(A_{p}=\right.$ $\left.\pi r^{2}\right)$.

Shear forces above a critical value of approximately $1.2 \times 10^{-6}$ dyne produce a filiform protrusion of the membrane ("tether") at the attachment site. The tether itself is not visible by light microscopy. The tether growth rate $\left(R_{T}, \mu \mathrm{m} / \mathrm{s}\right)$ is assessed by measuring the velocity by which the cell moves away from its attachment site. For each cell the tether growth rate $R_{T}$ was plotted against the shear force. The tether growth rate increases exponentially with increasing shear force (Fig. 1). The value of the critical shear force, $\mathrm{F}_{\mathrm{c}}$ (i.e. the shear force at which tether formation just begins), was determined from a 2 nd order exponential relationship established by least squares regression.

The plastic viscosity coefficient $\left(\eta_{\mathrm{P}}\right)$ was calculated from the following relationship $(23,24)$ :

$$
\eta_{\mathrm{P}}=\left(\mathrm{F}_{\mathrm{c}} / 8 \pi\right) \cdot\left(\mathrm{Gt} / \mathrm{R}_{\mathrm{T}}\right)
$$

where Gt is a dimensionless tether growth parameter (23) that is

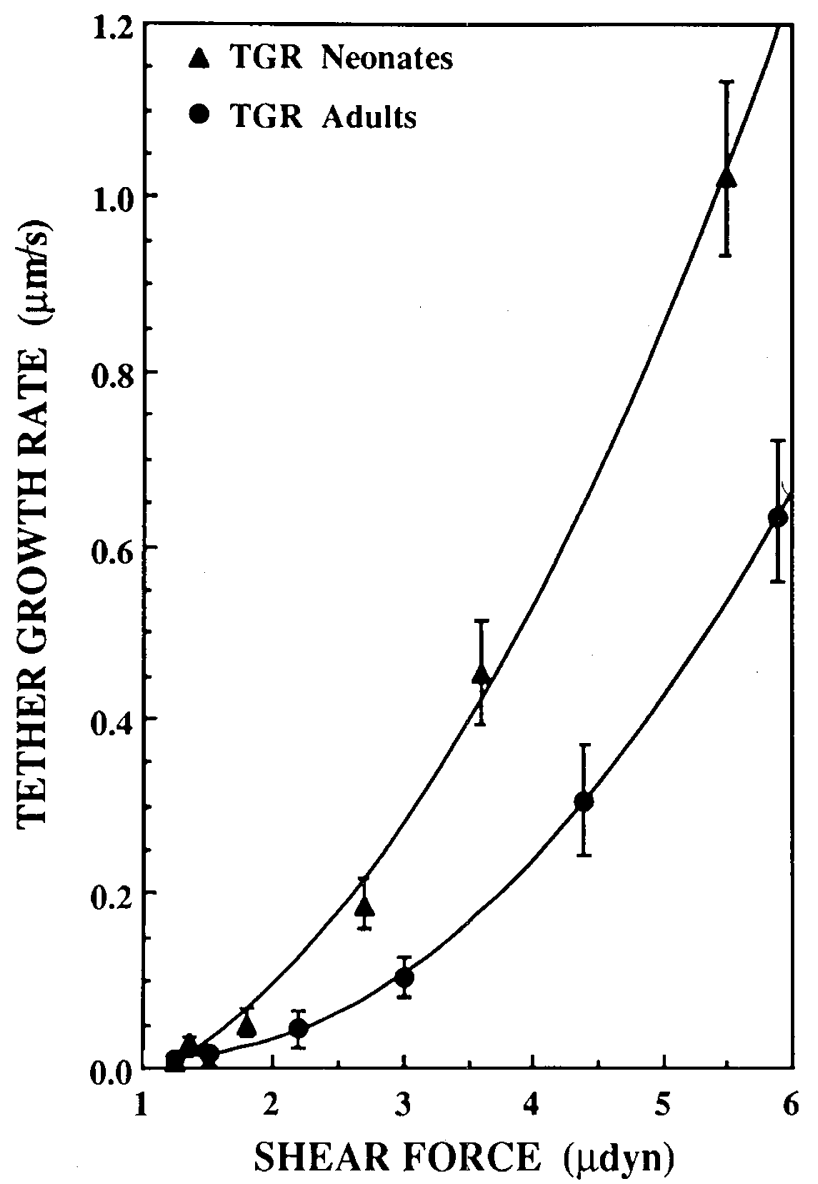

Fig. 1. Mean tether growth rate $(T G R)$ of point-attached neonatal and adult RBC plotted against the shear force in the flow channel. Vertical bars indicate the SD of TGR for individual neonatal and adult RBC. Exponential regression lines are fitted for both cell types. Note that the TGR of neonatal RBC was two times faster than that of adult RBC, whereas the critical shear force was similar for both cell types. 
a function of the ratio of $F_{s}$ to $F_{c}$. The value of $G t$ is $0,3.5,10.3$, and 18.6 when $F_{s} / F_{c}$ is $1,2,3$, and 4 , respectively $(23,24)$.

In one filling of the flow channel, only the cells under continuous observation can be studied because tethers may be pulled from all point-attached $\mathrm{RBC}$, thereby changing their membrane mechanical properties. Therefore, only three to six cells from each blood sample could be studied, resulting in a total number of 24 neonatal and 20 adult RBC available for final analysis.

Micropipette system. A micropipette and microscope-video system were used to determine the membrane extensional elastic modulus, membrane shear viscosity, and the tension for local membrane fragmentation of the same $\operatorname{RBC}(2,25)$. The microscope video system was that used for the flow channel. Details of the production and handling of micropipettes with inner diameters of 1.4-1.6 $\mu \mathrm{m} \mathrm{(26)} \mathrm{and} \mathrm{the} \mathrm{RBC} \mathrm{preparation} \mathrm{(15)} \mathrm{have}$ been described previously. One neonatal and one adult blood sample were studied on the same day, using the same micropipette; 28 to $33 \mathrm{RBC}$ were tested in each sample.

The time constant for extensional shape recovery was measured and analyzed by the method of Hochmuth et al. (27). The pipette was used to extend (overall extension ratio of approximately 1.6) and quickly release point-attached $\mathrm{RBC}$. From video recordings of the recovery process, the cell length-width ratio was measured at the first data point after releasing the cell and then every $1 / 50$ for 20 fields. The time constant was calculated as previously described (15).

The extensional elastic modulus and tension for local fragmentation were then studied after the extended cell had recovered its initial shape. The cell was detached from the coverslip, and a small membrane tongue was aspirated into the pipette from a flat portion of the cell surface $(18,25)$. The pipette pressure was increased in $1-\mathrm{mm} \mathrm{H}_{2} \mathrm{O}\left(98 \mathrm{dyne} / \mathrm{cm}^{2}\right)$ steps, and each pressure was held for periods of $10 \mathrm{~s}$. The pressure was increased until the membrane tongue ruptured (Fig. 2). The length of the membrane tongue $\left(\mathrm{L}_{\mathrm{T}}\right)$ in the pipette was measured at each pressure $(\mathrm{P})$ from video recordings. The extensional (shear) elastic modulus $\left(\mu_{\mathrm{e}}\right)$ was calculated from a linear relationship that exists between $\mathrm{L}_{\mathrm{T}} / \mathrm{R}_{\mathrm{p}}\left(\mathrm{R}_{\mathrm{p}}=\right.$ pipette radius) and $\mathbf{P} \cdot \mathrm{R}_{\mathrm{p}}$ over an $\mathrm{L}_{\mathrm{r}} / \mathrm{R}_{\mathrm{p}}$ range of approximately 1 to $3(25)$. Therefore, a linear approximation (3) to the theory developed by Evans and LaCelle (18) for analysis of this system was used:

$$
\left(\mathrm{P} \cdot \mathrm{R}_{\mathrm{p}} / \mu_{\mathrm{e}}=2.45\left(\mathrm{~L}_{\mathrm{T}} / \mathrm{R}_{\mathrm{p}}\right)-0.603\right.
$$

For a given pipette radius, $\mu_{\mathrm{e}}$ defines the pressure required to produce a defined membrane tongue length. In other words, $\mu_{\mathrm{e}}$ determines $\mathrm{RBC}$ membrane deformation in response to a given force. A small $\mu_{\mathrm{e}}$ indicates little resistance to deformation and high membrane flexibility. The membrane viscosity was calculated as the product of the time constant for extensional shape recovery and the extensional elastic modulus (27).

The stress resultant $\left(T_{S}\right)$ in the cylindrical section of the aspirated membrane tongue is $(25)$ :

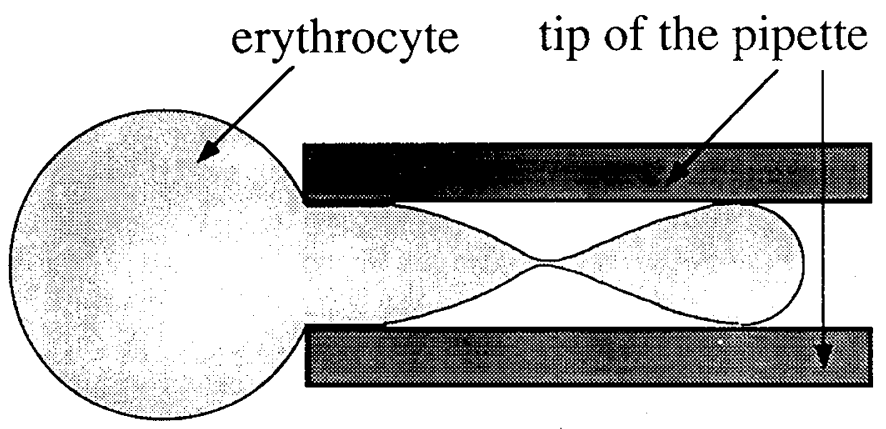

Fig. 2. Membrane failure of an $\mathrm{RBC}$ in a micropipette. The beginning of membrane failure defines the critical tension for local membrane fragmentation.

$$
\begin{array}{r}
\mathrm{T}_{\mathrm{S}}=\left(\mu_{\mathrm{e}} / 4\right)\left[\left(\mathrm{A}_{\mathrm{p}} / \mathrm{R}_{\mathrm{p}}^{2}\right)+\ln \left(1+\mathrm{A}_{\mathrm{p}} / \mathrm{R}_{\mathrm{p}}^{2}\right)\right] \mathrm{A}_{\mathrm{p}} \\
=\left(\mathrm{A}_{\text {cap }}+\mathrm{A}_{\text {cyl }}-\pi \mathrm{R}_{\mathrm{p}}^{2}\right) / \pi
\end{array}
$$

where $A_{\text {cap }}$ is the surface area of cap of the aspirated membrane tongue and $A_{c y l}$ is the surface area of the cylinder portion (Fig. 2).

$\mathrm{T}_{\mathrm{S}}$ at the yield point of the membrane was used as the tension for local fragmentation $\left(T_{F}\right)$. $T_{F}$ depends on both the extent of elastic membrane deformation and the stability of the membrane (25). Since $\mu_{\mathrm{e}}$ indicates membrane deformation at a given pressure, the effect of elastic membrane deformation can be minimized by calculating the dimensionless ratio $T_{F} / \mu_{\mathrm{e}}(25) . \mathrm{T}_{\mathrm{F}} / \mu_{\mathrm{e}}$ is proportional to $A_{p}$, which represents the extent of membrane deformation in the pipette. It is, therefore, a measure for the strain in the membrane.

Statistical analyses. Arithmetic means and SD were calculated for all RBC tested in each group. Thus, the SD reflect the spread of the individual $\mathrm{RBC}$ in each group and not of the donors. Differences between neonatal and adult $R B C$ were tested using the nonparametric, unpaired Wilcoxon rank sum test. Regressions were calculated to determine correlations between two parameters measured for the same RBC. Both linear and exponential functions were computed for each relationship. The functions that gave the best fits and highest correlation coefficients were selected. Differences between the slopes for neonatal and adult RBC were tested using a $t$ test.

\section{RESULTS}

Table 1 presents viscoelastic and viscoplastic material properties of neonatal and adult RBC membranes. The extensional elastic modulus of neonatal $\mathrm{RBC}$ was decreased compared with that of adult RBC, thereby indicating increased elastic deformability of RBC membrane in neonates. The membrane extensional viscosity was similar for both cell types.

The tension for local fragmentation was $23 \%$ lower in neonates than in adults. Thus, in a pipette with given dimensions, the aspiration pressure at which the RBC membrane ruptured was $23 \%$ less for neonates than for adults. However, the ratio of tension for local fragmentation to extensional (shear) elastic modulus (i.e. the strain in the membrane at the yield point) was only slightly increased for neonatal $\mathrm{RBC}(p>0.1)$. There were significant positive correlations between tension for local fragmentation and extensional (shear) elastic modulus for neonatal $(r=0.68)$ and adult $(r=0.57)$ RBC. The slopes for neonatal and adult $\mathrm{RBC}$ were not significantly different.

Membrane tether growth rates obtained for the neonatal and adult $\mathrm{RBC}$ over a wide range of shear forces are shown in Figure 1. Tether growth rate of neonatal RBC increased more with increasing shear force than that of adult cells $(p<0.05)$. The mean critical shear force was not different for both cell types. The critical wall shear stress (computed as the ratio of critical shear force and RBC surface area; see Materials and Methods) averaged 1.95 dyne $/ \mathrm{cm}^{2}$ for neonatal RBC and $2.38 \mathrm{dyne} / \mathrm{cm}^{2}$ for adult cells. This indicates that RBC membrane fragmentation of neonatal and adult RBC begins at similar shear forces, but that neonatal $\mathrm{RBC}$ membranes rupture more rapidly once the critical shear force is exceeded. The mean plastic viscosity coefficient calculated from the critical shear force and the tether growth rate was $50 \%$ less in the neonates than in adults (Table 1). Plastic viscosity coefficients ranged from 0.74 to $1.41 \times 10^{-2}$ dyne $\cdot \mathrm{s} / \mathrm{cm}$ in the neonates and from 1.56 to $2.33 \times 10^{-2}$ dyne . $\mathrm{s} / \mathrm{cm}$ in the adults.

\section{DISCUSSION}

We have assessed $\mathrm{RBC}$ membrane failure by means of a flow channel and a micropipette system. The critical shear force of membrane failure (i.e. beginning of tether formation) in the flow channel was similar for neonatal and adult RBC, whereas in the 
Table 1. Membrane mechanical properties (mean $\pm S D$ ) of neonatal and adult $R B C$

\begin{tabular}{lcc}
\hline & Neonatal RBC & Adult RBC \\
\hline Extensional elastic modulus $\left(10^{-3} \text { dyne } / \mathrm{cm}\right)^{*}$ & $5.0 \pm 0.7 \dagger$ & $6.1 \pm 0.8$ \\
Time constant for extensional recovery $(\mathrm{s})^{*}$ & $0.15 \pm 0.02 \dagger$ & $0.12 \pm 0.02$ \\
Extensional viscosity $\left(10^{-3} \text { dyne } \cdot \mathrm{s} / \mathrm{cm}\right)^{*}$ & $0.71 \pm 0.12$ & $0.75 \pm 0.14$ \\
Tension for local fragmentation $\left(10^{-3} \text { dyne } / \mathrm{cm}\right)^{*}$ & $31.1 \pm 3.7 \dagger$ & $40.2 \pm 5.0$ \\
$\mathrm{~T}_{\mathrm{F}} / \mu_{\mathrm{e}}^{*} \ddagger$ & $6.2 \pm 0.8$ & $6.6 \pm 0.9$ \\
Plastic viscosity coefficient $\left(10^{-2}\right.$ dyne.s $\left./ \mathrm{cm}\right) \S$ & $0.96 \pm 0.20 \dagger$ & $2.00 \pm 0.28$ \\
Critical shear force $\left(10^{-6}\right.$ dyne $) \S$ & $1.17 \pm 0.13$ & $1.19 \pm 0.21$ \\
\hline
\end{tabular}

* Data from $300 \mathrm{RBC}$ of 10 neonates and $300 \mathrm{RBC}$ of 10 healthy male adults.

$\dagger p<0.05$ when compared with adult $\mathrm{RBC}$ (Wilcoxon rank sum test).

$\$ T_{F} / \mu_{e}$, ratio of tension for local fragmentation to extensional elastic modulus.

$\S$ Data from $24 \mathrm{RBC}$ of six neonates and $20 \mathrm{RBC}$ of five healthy male adults.

micropipette membrane failure of neonatal $\mathrm{RBC}$ occurred at smaller shear forces compared with adult RBC. The apparent discrepancy of these results can be explained by different effects of the applied forces on the membrane material, which depend on the resulting strain in the membrane. The strain cannot be directly measured; it can only be estimated from the extent of membrane deformation $(18,19)$.

In the micropipette, the RBC membrane deforms markedly before it ruptures. Because of the smaller extensional elastic modulus, the membrane of neonatal RBC deforms more in response to a given force than that of adult $\mathrm{RBC}$. This explains why the membrane tongue length in the pipette was about $7 \mu \mathrm{m}$ at the yield point for both neonatal and adult RBC although the critical aspiration pressure was lower for neonatal cells. Thus, the membrane of neonatal and adult $\mathrm{RBC}$ ruptured at different pressures but at similar extents of membrane deformation (i.e. at similar strains).

In the flow channel, the yield point is defined as the critical shear force at which a membrane tether begins to form. Tether formation results from the failure of a tiny membrane area without fragmentation of the membrane. The critical shear force is independent of membrane deformation (27), because the strain depends only on the force acting on the attached membrane point. We therefore conclude that both the flow channel and the micropipette results indicate similar mechanical stability of neonatal and adult RBC when the different elastic membrane deformabilities are considered for the micropipette experiments.

The flow channel experiments also showed that the tether formation of neonatal RBC membrane develops two times faster compared to adult RBC. This is reflected by the markedly lower plastic viscosity coefficient of neonatal RBC (Table 1).

The mechanical membrane properties are mainly determined by the membrane protein skeleton (28). The membrane skeleton consists of spectrin, actin, tropomyosin, and proteins 4.1 and 4.9. Membrane stability appears to be mainly influenced by the spectrin self-association and by interactions between spectrin, actin, and band 4.1 (28). Waugh and Agre (29) showed that RBC membrane shear elasticity in hereditary spherocytosis depends on the surface density of spectrin in the cell membrane. Molecular defects in a stress-supporting region of the membrane were found to reduce the critical force for membrane failure, whereas specific molecular defects do not appear to affect the plastic membrane viscosity coefficient (30). Our results indicate that the structural peculiarities leading to alterations in the membrane mechanics of neonatal RBC are different from those in hereditary spherocytosis, inasmuch as neonatal RBC showed no significant reduction in the critical shear force (Table 1).

The biochemical basis for the different membrane mechanical properties of neonatal RBC is unclear. Neonatal and adult RBC show no qualitative or gross quantitative differences in the pattern of the membrane proteins $(31,32)$. Lane et al. (8) observed increased membrane-associated $\mathrm{Hb}$ in senescent neonatal $\mathrm{RBC}$. Increased membrane-bound $\mathrm{Hb}$ is associated with increased membrane shear viscosity but does not alter membrane elasticity (33). Landaw et al. (34) reported reduced protein extraction from neonatal RBC ghosts and concluded that the spectrin lattice is more tightly linked in resealed membranes when compared with adult RBC. Stability of intact RBC membrane may be influenced by intracellular components that regulate protein interactions in the membrane. In particular, 2,3-diphosphoglycerate may dissociate spectrin-actin-protein 4.1 complexes, thereby decreasing membrane stability (28). However, in neonates, intraerythrocytic 2,3-diphosphoglycerate is markedly lower than in adults (35). Recently, the contribution of the band 3-ankyrin interaction to $\mathrm{RBC}$ membrane mechanical stability of normal human $\mathrm{RBC}$ was described (36). Similar studies have not been done on neonatal $\mathrm{RBC}$.

The question arises whether the models and shear forces used in our study are comparable to in vivo conditions. In vivo, the wall shear stress (i.e. the greatest shear stress in a vessel) reaches its maximum (approximately 100 dyne $/ \mathrm{cm}^{2}$ ) in capillaries (37). Hemolysis of freely flowing RBC requires a shear stress of 1500 to 2500 dyne $/ \mathrm{cm}^{2}$ (38). Lower shear stresses for membrane rupture are necessary when the exposure time to the shear forces is prolonged (39) because of cell trapping and sticking. The micropipette method provides a model for RBC trapping in capillaries. The flow channel can be regarded as a model for RBC sticking to endothelium. Membrane rupture in the micropipette occurred at a shear stress of about $100 \mathrm{dyne} / \mathrm{cm}^{2}$. Point-attached $\mathrm{RBC}$ (flow channel) yielded at a shear stress of approximately 2 dyne $/ \mathrm{cm}^{2}$. The extremely low critical yield shear stress in the flow channel may be explained by the small attachment area of the cells (39). Because the wall shear stress in capillaries reaches the yield shear stress in micropipettes and exceeds the critical shear stress for point-attached RBC, we believe that our methods provide reasonable models for in vivo $\mathrm{RBC}$ fragmentation.

We conclude that the membrane of neonatal RBC deforms more in response to a given shear force than does that of adult $\mathrm{RBC}$. This results in larger strain and greater susceptibility of the neonatal RBC membrane to yield and fragment. Moreover, the faster tether growth suggests that neonatal RBC lose more membrane per unit time once the yield shear force has been exceeded. These peculiar membrane mechanical properties of neonatal RBC may contribute to their accelerated membrane loss (6) and shortened life span (1).

\section{REFERENCES}

1. Bratteby LE, Garby L, Groth T, Schneider W, Wadman B 1968 Studies on erythro-kinetics in infancy. XII. The mean life span and the life span frequency function of red blood cells formed during foetal life. Acta Paediatr Scand 57:311-320

2. Linderkamp O, Meiselman HJ 1982 Geometric, osmotic, and membrane mechanical properties of density-separated human red cells. Blood 59:11211127

3. Nash GH, Meiselman HJ 1981 Red cell ageing: changes in deformability and other possible determinants of in vivo survival. Microcirculation 1:255-284

4. Clark MR 1988 Senescence of red blood cells: progress and problems. Physiol Rev 68:503-554

5. Linderkamp O, Wu PYK, Meiselman HJ 1982 Deformability of density separated red blood cells in normal newborn infants and adults. Pediatr Res 16:964-968 
6. Matovcik LM, Chiu D, Lubin B, Mentzer WC, Lane PA, Mohandas N, Schrier SL 1986 The aging process of human neonatal erythrocytes. Pediatr Res 20:1091-1096

7. Komazawa M, Oski FA 1975 Biochemical characteristics of "young" and "old" erythrocytes of the newborn infant. J Pediatr 87:102-106

8. Lane PA, Galili U, Iarocci TA, Shew RA, Mentzer WC 1988 Cellular dehydration and immunoglobulin binding in senescent neonatal erythrocytes. Pediatr Res 23:288-292

9. Kleihauer E, Bernau A, Betke K, Keller M, Kohne E, Riegel K 1970 Heinzkörperbildung in Neugeborenenerythrozyten. I. In vitro Studien über experimentelle Bedingungen und den Einfluß von Austauschtransfusionen. Acta Haematol 43:333-347

10. Bracci R, Martini G, Buonocore G, Talluri B, Berni S, Ottaviani MF, Picchi MP, Casini A 1988 Changes in erythrocyte properties during the first hours of life: electron spin resonance of reacting sulfhydryl groups. Pediatr Res 24:391-395

11. Matovcik LM, Junga IG, Schrier SL 1985 Drug-induced endocytosis of neonatal erythrocytes. Blood 5:1056-1063

12. Sjölin S 1954 The resistance of red cells in vitro. A study of the osmotic properties, the mechanical resistance and the storage behaviour of red cells of fetuses, children and adults. Acta Paediatr 43(suppl 98):1-92

13. Goldbloom A, Fischer E, Reinhold J, Hsia DY 1953 Studies on the mechanical fragility of erythrocytes. I. Normal values for infants and children. Blood 8:165-169

14. Tarssanen L 1976 Hemolysis by ultrasound. Scand J Haematol Suppl 29:1-58

15. Linderkamp O, Nash GH, Wu PYK, Meiselman HJ 1986 Deformability and intrinsic material properties of neonatal red blood cells. Blood 67:1244-1250

16. Danon Y, Kleimann A, Danon D 1970 The osmotic fragility and density distribution of erythrocytes in the newborn. Acta Haematol 43:242-247

17. Linderkamp O, Meiselman HJ, Wu PYK, Miller FC 1981 Blood and plasma viscosity and optimal hematocrit in the normal newborn infant. Clin $\mathrm{He}$ morheol 1:575-584

18. Evans EA, LaCelle PL 1975 Intrinsic material properties of the erythrocyte membrane indicated by mechanical analysis of deformation. Blood 45:2943

19. LaCelle PL, Evans EA, Hochmuth RM 1977 Erythrocyte membrane elasticity, fragmentation, and lysis. Blood Cells 3:335-350

20. Riegel K, Linderkamp O 1991 Das Neugeborene. In: Betke K, Künzer W, Schaub J (eds) Keller/Wiskott Lehrbuch der Kinderheilkunde. Thieme, Stuttgart, pp 159-170

21. Hochmuth RM, Mohandas N, Blackshear PL 1973 Measurement of the elastic modulus for red cell membrane using a fluid mechanical technique. Biophys J 13:747-762
22. Waugh RE 1982 Temperature dependence of the yield shear resultant and the plastic viscosity coefficient of the erythrocyte membrane. Biophys J 39:273278

23. Evans EA, Hochmuth RM 19.76 Membrane viscoplastic flow. Biophys J 16:13 26

24. Hochmuth RM, Evans EA, Colvard DF 1976 Viscosity of human red cell membrane in plastic flow. Microvasc Res 11:155-159

25. Evans EA 1973 New membrane concept applied to the analysis of fluid shear and micropipette deformed red blood cells. Biophys J 13:941-954

26. Weiss T, Ruef P, Linderkamp O 1991 A simplified method to produce micropipettes with flat tips. Clin Hemorheol 11:383-386

27. Hochmuth RM, Worthy PR, Evans EA 1979 Red cell extensional recovery and the determination of membrane viscosity. Biophys J 26:101-114

28. Chasis JA, Mohandas N 1986 Erythrocyte membrane deformability and stability: two distinct membrane properties that are independently regulated by skeletal protein associations. J Cell Biol 103:343-350

29. Waugh RE, Agre P 1988 Reductions of erythrocyte membrane viscoelastic coefficients reflect spectrin deficiencies in hereditary spherocytosis. J Clin Invest 81:133-141

30. Waugh RE 1983 Effects of abnormal cytoskeletal structure on erythrocyte membrane mechanical properties. Cell Motil 3:609-622

31. Shapiro DL, Pasqualini P 1978 Erythrocyte membrane proteins of premature and full-term newborn infants. Pediatr Res 12:176-178

32. Lawler J, Liu SC, Palek J, Prchal J 1984 A molecular defect of spectrin in a subset of patients with hereditary elliptocytosis. Alterations in the a subunit domain involved in spectrin self-association. J Clin Invest 73:1688-1695

33. Friedrichs E, Meiselman HJ 1990 Calcium regulated membrane-attached hemoglobin and mechanical properties of density-fractionated erythrocyte membranes: implications for hemolytic diseases and RBC aging. Pediatr Res 28:299(abstr)

34. Landaw SA, Rathbun SC, Guancial RL 1982 Evidence for a tightly-linked spectrin lattice in red blood cells of newborn man. Pediatr Res 16:208(abstr)

35. Versmold HT, Linderkamp O, Döhlemann C, Riegel KP 1976 Oxygen transport in congenital heart disease: influence of fetal hemoglobin, red cell $\mathrm{pH}$ and 2,3-diphosphoglycerate. Pediatr Res 10:566-570

36. Low PS, Willardson BM, Mohandas N, Rossi M, Shohet S 1991 Contribution of the band 3-ankyrin interaction to erythrocyte membrane mechanical stability. Blood 77:1581-1586

37. Schmid-Schönbein H 1976 Blood flow and erythrocyte distribution in the microcirculation. Int Rev Physiol 9:1-62

38. Sutera SP 1977 Flow-induced trauma to blood cells. Circ Res 41:2-8

39. Evans E, Berk D, Leung A 1991 Detachment of agglutinin-bonded red blood cells. I. Forces to rupture molecular-point attachments. Biophys J 59:838848 